\title{
Statistics of South Delta-Aquariids (SDA) meteor showers registered at UNIVAP in 2017 and 2018
}

\author{
Rita de Cássia Alves da Silva ${ }^{1}$, Guilherme Jayme Allan Pimentel ${ }^{2}$, Francisco Carlos Rocha \\ Fernandes $^{2}$, Lucas Antonio Caritá ${ }^{*}$, Irapuan Rodrigues ${ }^{2}$ \\ ${ }^{1}$ Instituto Federal de Educação, Ciência e Tecnologia de São Paulo, São José dos Campos, SP, Brasil \\ ${ }^{2}$ Universidade do Vale do Paraíba, São José dos Campos, SP, Brasil
}

Received on January 1, 2019. Revised on April 22, 2019. Accepted on May 27, 2019.

\begin{abstract}
Meteor showers, seen at regular and frequent intervals, occur when the Earth crosses the orbit of a comet and many small dust particles enter the Earth's atmosphere. In this paper we compare the Southern Delta-Aquariids (SDA) shower recorded by the UVP1 and UVP2 meteor monitoring stations in the Southeastern of Brazil, in 2017 and 2018. These stations are affiliated with EXOSS Citizen Science meteor monitoring network. The SDA meteor shower represented the most active radiant in these two years of data recorded by these stations. 27 detections were achieved in 2017 (between July 12 and August 24) and 15 detections in 2018 (between July 18 and August 20). The meteor properties highlighted and compared throughout the present text are: duration, apparent magnitude and angular velocity. We used estimations provided by the UFOAnalyzer program, since there is no doubled captures from diverse stations far separated. This article helps to highlight the importance of the EXOSS project, aligned with the concept of citizen science, valuing the partnership between amateur astronomers and professional scientists in data collection and wide dissemination of knowledge related to the science of meteors. This work is the result of two scientific initiation projects, with an educational cooperation between the Universidade do Vale do Paraíba (UNIVAP) and the Instituto Federal de Educação, Ciência e Tecnologia de São Paulo campus São José dos Campos (IFSP-SJC).
\end{abstract}

Keywords: Meteor shower, South Delta Aquariids, EXOSS.

\section{Introduction}

Meteoroids are solid spatial particles large enough to produce light caused by the ablation during their passage through the Earth's atmosphere 1]. This lightening phenomenon is called meteor. Such particles are small fragments of comets or asteroids and usually burn completely before reaching the ground [2].

At every passage near the Sun, a comet produces and deposits, along its orbit, a large amount of dust particles. When the Earth crosses the orbit of a comet and many of these particles enter the Earth's atmosphere, the so-called meteor shower occurs. The radiant of a meteor is the point on the celestial sphere from which the meteor appears. They are best observed in meteor showers, where several meteors seem to come from a single region of the sky (the radiant) 2. Only $25 \%$ of the meteors are associated to a meteor shower 1 .

Meteor showers, seen at regular and frequent intervals, may occur when the Earth passes through bundles of meteoroids that can be produced by the decay of comets along their orbits, especially when they are in the perihelion 3, 4. The Earth daily finds in its path several bundles of meteoroids [1]. According to [2], these bundles, for the most part, have little known orbits and their

* Correspondence email address: prof.carita@ifsp.edu.br particles can be confused with sporadic meteoroids. However, there are well-defined bundles that are associated with so-called "large meteor showers" These showers may also occur by the fragmentation resulting from collisions suffered by asteroids whose highest efficiency occurs in aphelion [5] or, also, due to the breakdown of comet nuclei $[6]$.

Meteor showers receive the name of the constellation where the radiant is located. According to [7, the Southern Delta-Aquariids (SDA) shower was detected for the first time in 1849. The activity period of the SDA shower extends from July 8 to August 19, usually showing a maximum peak of activity around July 29 and 30 [7], with its radiant located about 3 degrees West of the star Skat ( $\delta$ of the Aquarium Constellation) 8, 9. It also has an apparent magnitude of 3.310 12. The meteor velocities in the SDA are approximately $41 \mathrm{~km} / \mathrm{s}[13$. According to 14, the SDA meteor shower is rich in low-intensity meteors. However, brighter Delta-Aquariids may occur, especially in the Southern Hemisphere, because the radiant is high enough in the sky, allowing the observation from eventide.

According to 15, there is still controversy over the parental body of the radiant SDA. Debris from SDA meteors originate from the Mardsen Group, a class of comets known as sungrazing, because they have orbits 
whose perihelion is very close to the Sun [16]. According to NASA's website [13], comet 96P/Machholz has recently emerged as a possible parental body for the space debris that spawns SDA meteors. Discovered in 1986, Comet $96 \mathrm{P} /$ Machholz has a core about 6.4 kilometers long and has a short orbital period of 5.2 years. Its orbit has aphelion (maximum distance from the Sun) beyond the orbit of Jupiter, and perihelion (closest approach to the Sun), more internal than the orbit of Mercury. However, in an extensive study of parental bodies of several known meteor showers performed by [17, no parental body was found for the Southern Delta-Aquariids.

This work aims to compare the SDA meteors registered by the UVP1 and UVP2 meteor monitoring stations in 2017 and 2018, since this radiant presented the highest numbers of meteors recorded by both stations in these two years. The stations UVP1 and UVP2 are located at the Universidade do Vale do Paraíba (UNIVAP), in Brazil (city of São José dos Campos - São Paulo state) and they are affiliated to the EXOSS (Exploring the Southern Sky) monitoring stations network (http://press.exoss.org/).

The present work is the result of two scientific initiation projects, with an educational cooperation between the Universidade do Vale do Paraíba (UNIVAP) and the Instituto Federal de Educação, Ciência e Tecnologia de São Paulo campus São José dos Campos (IFSP-SJC).

\section{Methodology}

EXOSS is a collaborative citizen science network, working together with several scientific institutions, focused on the study of meteors and bolides, their origins, natures and characterization of their orbits. EXOSS started its activities in May 2015, with 9 associates and with rapid expansion. Currently, the EXOSS network has 58 associates (17 institutions) in 15 Brazilian states, operating 78 cameras daily in meteor records and data collection for the study of their dynamics and nature. The network accepts collaboration from professional and amateur astronomers. It aims to record, catalog and promote astrometric and astrophysical meteor studies. In this sense, EXOSS stations monitor the sky daily by recording videos of the captured events, always valuing the partnership between amateur astronomers and professional scientists in data collection and wide dissemination of knowledge related to the science of the meteors.

The EXOSS affiliated UVP1 and UVP2 monitoring stations were installed in January 2017 and September 2017, respectively, in UNIVAP campus, located in São José dos Campos, São Paulo, Brazil, (23.21 S latitude, $45.96^{\circ} \mathrm{W}$ longitude). Each monitoring station operates in conjunction with a camera, which every night detects the meteors in a fixed region of the sky. UVP1 points to the azimuth $138^{\circ}$, elevation $63^{\circ}$, and has a field of view of $64.25^{\circ}$. UVP2 points to the azimuth $63^{\circ}$, elevation $58^{\circ}$, and has a field of view of $88.62^{\circ}$. Figure 1 shows the location and direction of view of stations UVP1 and UVP2. They have only approximately $1.4^{\circ}$ of field of view superposition, being difficult to occur simultaneous meteor detections. As part of the EXOSS network, each station is able to triangulate with two other cameras in nearby cities, to perform orbit determinations.

UFOCapture and UFOAnalyzer are the softwares ${ }^{1}$ used for recording and analyzing data at EXOSS stations. The UFOCapture software automatically records a video from the sky whenever a light phenomenon is captured by the camera. The UFOAnalyzer software allows to determine the parameters and properties of the detected meteors, such as probable radiant, apparent magnitude, duration and angular and linear velocities. Thus, from the data recorded by these softwares, the radiant with the greatest number of detected events was identified for the years 2017 and 2018 in the two stations and, using automatic software tools, the main properties of the meteors associated to this radiant were estimated.

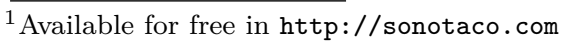

UVP-1 | Univap | $a z=138^{\circ}, \mathrm{ev}=63^{\circ}, \mathrm{fov}=64^{\circ}, \mathrm{d}=158 \mathrm{Km}, \mathrm{d} 2=190 \mathrm{Km}$

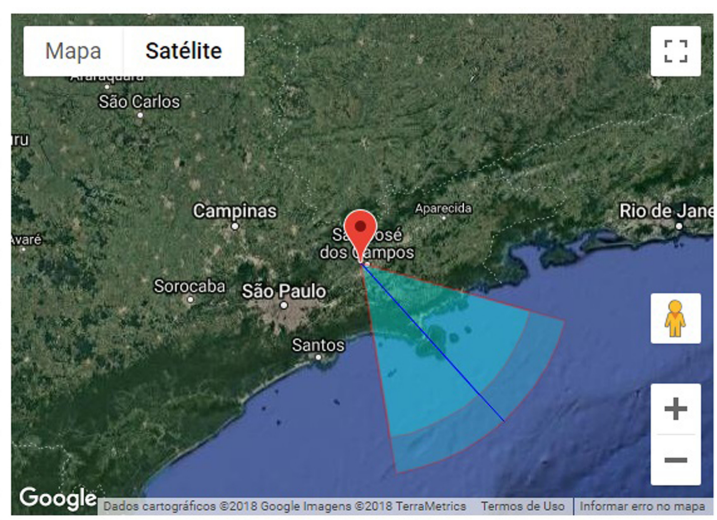

UVP-2 | Univap $\mid \mathrm{az}=63^{\circ}, \mathrm{ev}=58^{\circ}, \mathrm{fov}=88^{\circ}, \mathrm{d}=199 \mathrm{Km}, \mathrm{d} 2=250 \mathrm{Km}$

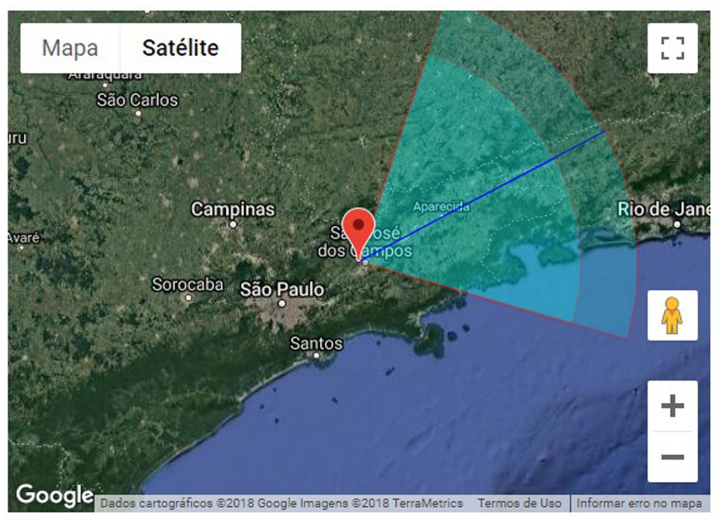

Figure 1: UNIVAP stations coverage area: UVP1 (left) and UVP2 (right). Source: https://live.exoss.org/ (Accessed December $28,2018)$. 


\section{Results and Discussions}

Figure 2 shows the daily distribution of SDA, the main meteor shower recorded by UVP1 and UVP2 stations in 2017 (27 detections between July 12 and August 24) and in 2018 (15 detections between July 18 and August $20)$. There were no double detections between stations UVP1 and UVP2. These numbers pointed to a decrease of almost $50 \%$ in the number of meteors registered between 2017 and 2018. However, in 2017, only the station UVP1 was in operation.

The highest activity was recorded on July 27, for the year 2017 and on July 29, for 2018. These results are in agreement with the maximum peak of activity reported by 7 .

Figure 3 shows all SDA meteors detected by stations UVP1 and UVP2 in 2017 and 2018.

The properties (duration, apparent magnitude and angular velocity) of SDA meteors for 2017 and 2018 are discussed in the next sections.

\subsection{Duration}

In the UVP detections, the duration ranged from 0.06 to 0.44 seconds, in 2017 and from 0.16 to 0.60 seconds, in 2018, with mean values of 0.18 and 0.27 seconds, respectively. See Figure 4

\subsection{Magnitude}

The UFOAnalyzer software measures object's magnitude by the comparison with stars based on color temperature. The apparent magnitude of SDA meteors recorded by UNIVAP's stations ranged from 0.0 to -2.6 in 2017 and from -0.5 to -3.5 in 2018 , with mean values of -1.1 and -1.4 , respectively. The distribution of the apparent magnitudes determined is shown in Figure 5

Most SDA meteors are of low intensity (only about $30 \%$ had a magnitude lower than -1.47 , the magnitude of the star Sirius), agreeing with [14] and, thus, reinforcing the idea of penetration in high altitudes.

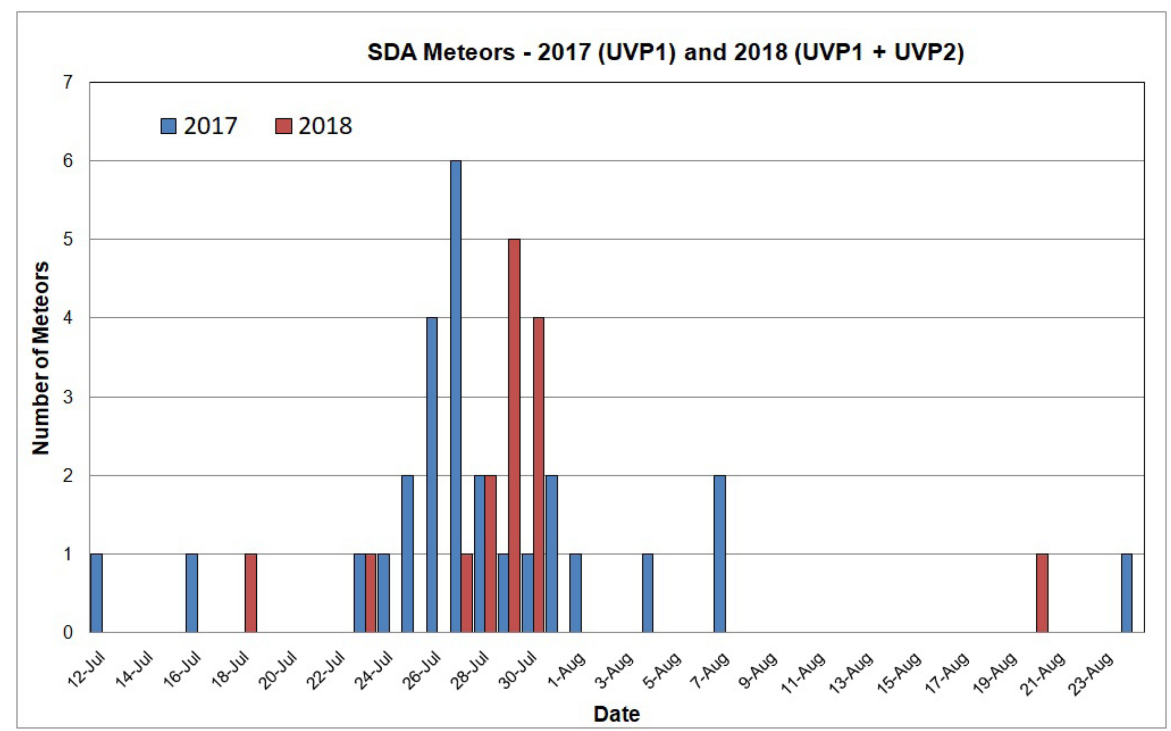

Figure 2: Daily distribution of SDA detections from July 12 to August 24 for the years 2017 (only station UVP1) and 2018 (stations UVP1 and UVP2).

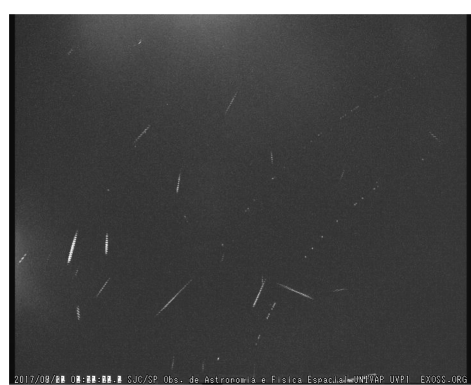

(a)

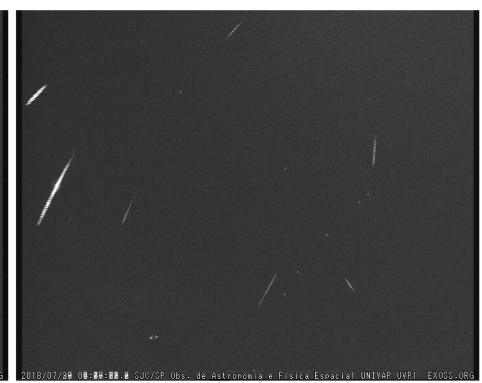

(b)

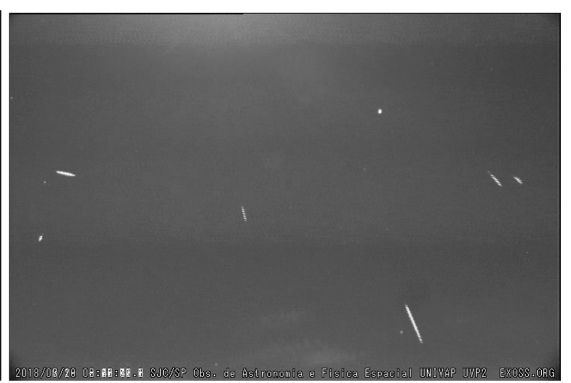

(c)

Figure 3: Composition images for SDA meteors captured by UNIVAP Stations: (a) 27 SDA captures from July 12, 2017 to August 24, 2017 (station UVP1); (b) 7 SDA captures between July 23, 2018 and July 30, 2018 (station UVP1); and (c) 6 SDA captures from July 18, 2018 to August 20, 2018 (station UVP2). Two images were not included in the last composition due to the presence of the moon. 


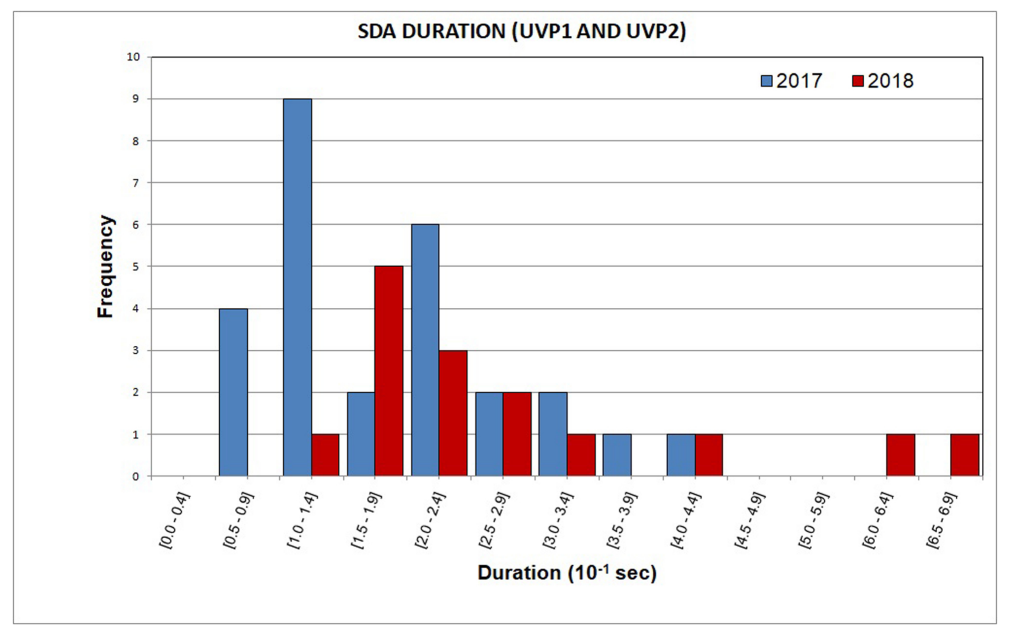

Figure 4: Histogram of the distribution of SDA meteor duration for 2017 and 2018.

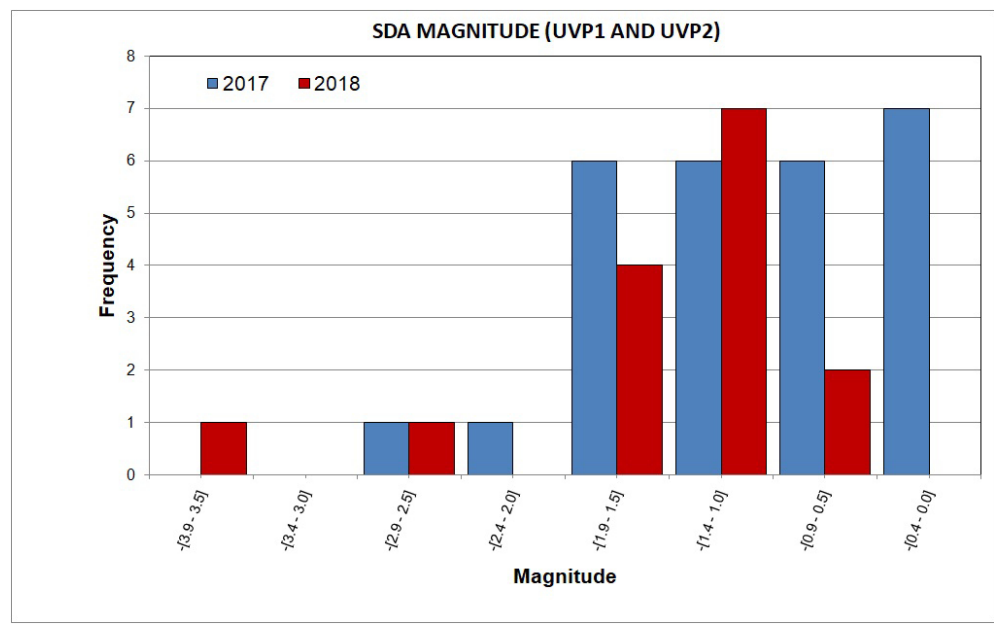

Figure 5: Magnitude distribution of SDA meteors for 2017 and 2018.

\subsection{Angular Velocity}

The angular velocity determined ranged between 3.7 and 21.3 degree/s, for meteors captured in 2017, with an average of 13.9 degree/s and between 9.1 and 20.6 degree/s, for 2018, with a mean value of 14.8 degree/s. See Figure 6

Besides angular velocity, the linear velocity was also automatically estimated by UFOAnalyzer. The estimated values of velocity are between 27.4 and $56.3 \mathrm{~km} / \mathrm{s}$, with an average of $47.1 \mathrm{~km} / \mathrm{s}$ and between 47.3 and $56.2 \mathrm{~km} / \mathrm{s}$, with a mean value of $48.7 \mathrm{~km} / \mathrm{s}$, for 2017 and 2018, respectively.

The average velocities estimated $(47.1 \mathrm{~km} / \mathrm{s}$ and 48.7 $\mathrm{km} / \mathrm{s}$ ) for the SDA meteors recorded by stations UVP1 and UVP2 are higher than the typical $41 \mathrm{~km} / \mathrm{s}$ indicated by 13$]$.

The precise determination of the meteor trajectory, including its velocity, is only possible when simultaneous detections occur for two or more stations (pairing), which are separated by a distance of tens of kilometers, allowing a triangulation between the records of the different sta- tions and calculation of the meteor trajectory parameters, which does not occur for stations UVP1 and UVP2.

In the case of detections (captures) by only one station, as in the present analysis, the automatic velocity determination by the UFOAnalyzer is just an estimation that depends on the assumption of certain parameters, such as the height of the beginning of the trail, considered for each meteor shower.

Although less frequent in 2018, the SDA meteors were, on average, brighter, longer-lasting, and faster than those recorded in 2017.

It is worth mentioning that the velocities of entry of the meteors are between $11.2 \mathrm{~km} / \mathrm{s}$ and $72.8 \mathrm{~km} / \mathrm{s}$, values preestablished by 11. The lowest velocity value is due to the gravitational pull of the meteoroids, while the greatest value is due to the composition of the escape velocity of the solar system with the Earth's orbital velocity.

According to [1], most meteors occur in the thermosphere, between 80 and $90 \mathrm{~km}$ of altitude, but faster meteors may become visible above this height. The results of [2] show that SDA recorded in 1999, 2000 and 2001 occurred between 85 and $93 \mathrm{~km}$ of altitude. There- 


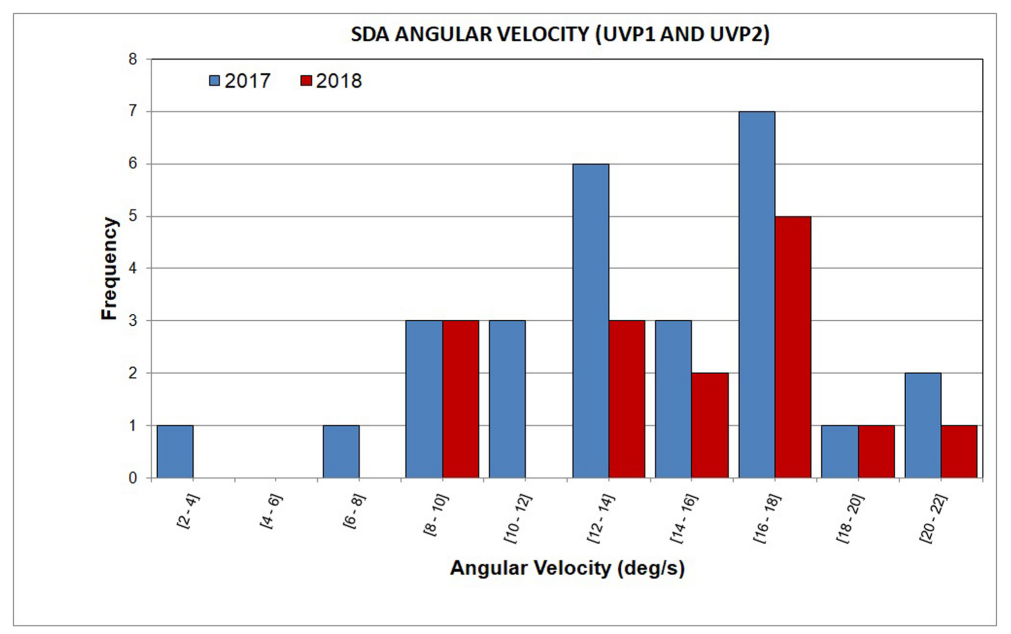

Figure 6: Angular Velocity distribution of SDA meteors for 2017 and 2018.

fore, the velocity results suggest that SDA meteors may have penetrated the higher layers in the atmosphere.

\section{Conclusions}

In this work, the properties of the meteors associated to the Southern Delta-Aquariids meteor shower, registered in 2017 and 2018, by the stations UVP1 and UVP2 installed at UNIVAP (which are part of the EXOSS meteor monitoring network), were compared. This meteor shower represented the most active radiant in these two years, probably due to the SDA meteor shower being observed in the Southern Hemisphere during the winter, when the sky is generally cleaner and without moisture.

It is important to highlight the importance of the EXOSS project, in line with the concept of citizen science, valuing the partnership between amateur astronomers and professional scientists in data collection and wide dissemination of knowledge related to the science of meteors.

\section{Acknowledgments}

R. C. A. Silva and G. J. A. Pimentel are grateful to CNPq for the award of PIBIC and PIBITI grants for scientific initiation at the institutions UNIVAP and IFSP, respectively. F. C. R. Fernandes and I. Rodrigues acknowlege receiving PQ-CNPq scholaship (Processess 311376/20150 and 311920/2015-2, respectively). F. C. R. Fernandes also acknowledges FAPESP for Regular Project (Process 2017/02806-3). The authors also appreciate all the support offered by the EXOSS team.

\section{References}

[1] Z. Ceplecha, J. Borovička, W.G. Elford, D.O. ReVelle, R.L. Hawkes, V. Porubčan and M. Šimek, Space Science Reviews 84, 327 (1998).
[2] J.A.S. Corrêa, Estudo de meteoros e investigações de seus efeitos na ionosfera com dados do radar SKiYMET e GPS. Master Thesis, INPE, São José dos Campos, (2013).

[3] F.L. Whipple, in: An Isothermal Atmosphere, Proceedings of the National Academy of Science (National Academy of Science, Washington, 1950).

[4] F.L. Whipple, Astrophysical Journal 113, 464 (1951).

[5] I.P. Williams, in: Meteoroids and their Parent Bodies edited by J. Stohl and I.P. Williams (Slovak Academy of Sciences, Bratislava, 1993), p. 31.

[6] D.I. Steel, D.J. Asher, S.V.M. Clube, Monthly Notices of the Royal Astronomical Society, 251, 632 (1991).

[7] R. Cardona, J.N. Madiedo and J.M. Trigo-Rodríguez, in: 44th Lunar and Planetary Science Conference (Lunar and Planetary Institute; Universities Space Research Association, The Woodlands, 2013), available in http:// www.lpi.usra.edu/meetings/lpsc2013/pdf/1122.pdf

[8] Tudo sobre a chuva de meteoros Delta Aquaridas Austrais, available in http://www.galeriadometeorito com/2018/07/chuva-de-meteoros-delta-aquaridasaustrais-2018.html.

[9] Skat, Delta Aquarii, 76 Aquarii, available in http://www universeguide.com/star/skat.

[10] Delta Aquariids (Aquarids) meteor showers peak on July 28th / 29th, 2015, available in http: //freestarcharts.com/delta-aquariids-aquaridsmeteor-showers-peak-on-july-28th-29th-2015.

[11] Delta Aquariids Kick Off Summer Meteor Showers, available in http://www.skyandtelescope.com/astronomynews/observing-news/delta-aquarids-kick-offsummer-meteor-showers.

[12] Meteor Shower Basics, available in http: //www .amsmeteors.org/meteor-showers/meteorshower-basics

[13] Delta Aquariids, available in http://solarsystem nasa.gov/small-bodies/meteors-and-meteorites/ delta-aquariids.

[14] Royal Astronomical Society of New Zeland. Table of Southern Meteor Showers. Web Site; http://rasnz.org.nz/in-the-sky/meteor-showers\#list, 2018 . 
[15] P. Jenniskens, in: Meteor Showers and their Parent Comets (Cambridge, University Press, 2006).

[16] A chuva de meteoros campeã no Brasil é..., available in http://http://press.exoss.org/a-chuva-demeteoros-campea-no-brasil-e/

[17] T.J. Jopek, Memorie della Societa Astronomica Italiana 82, 310 (2014). 